\title{
Stochastic Differential Game Analyses of Tailored Taxi Service Coordination by Cost Sharing Contract
}

\author{
Haifeng Zhao ${ }^{a}$, Dan Chen ${ }^{b}$ \\ School of Economics and Management, Tongji University, Shanghai 200092, China \\ azhfgroup@163.com, bdannahere@163.com
}

\begin{abstract}
Keywords: Tailored Taxi Service; System Coordination; Cost Sharing; Stochastic Differential Game.
\end{abstract}

\begin{abstract}
Cooperation of all the members in the tailored taxi service plays an important role in the development of this fast-developing service industry. The platform enterprise can encourage cooperation from special car drivers by sharing their cost during the service, which we assume can increase the sales by accumulating the reputation of the service model. A differential game model is established with tailored taxi service that consists of one platform enterprise and one special car driver and we derive the optimal solutions of the Stackelberg equilibrium with the platform enterprise as the leader who partially shares the cost of the efforts of the driver and the cooperative game that both layers make efforts together to get the most profits of this system. The results make the benefits of the cost sharing contract in increasing the profits of both players as well as the whole service system, which means that the cost sharing contract is an effective coordination mechanism in the long-term relationship of the members in the service system.
\end{abstract}

\section{Introduction}

In recent years, as the increasing traffic pressure of our national cities, government's policies of limited traffic are adopted in succession, the rhythm of people's life speed up, more and more people start to select the flexible, convenient and rapid taxi taking way to go out. But the serious mismatching information between the taxi drivers and the passengers cause a lot of cases frequently, like the taxes emptied load in non-rush hour, the drivers refusing to take on rush hour and severe weather, or the taxi's demand exceeding the supply, difficult to taking a taxi disturb the inhabitant' daily trip. Meanwhile, many social resource like personal car, are not allowed to developed and utilized, which cause the resource waste on the condition that many passengers' demand for the taxi are not meet.

Under the current situation, in order to achieve more efficient travel, tailored taxi service and related apps come out. Through the establishment of the information communication platform between the car drivers and users, this service system improves the success rate of taking a taxi, the driving experience of passengers, and achieve the double-win to both the car drivers and users, which have become more and more popular in many cities. Taking platform enterprises represented by Uber, Didi and Kuaidi, with their advanced operating technology and flexible mode of commercial operation, win lots of praise under the background of rapid development of network technology in our country and intelligent terminal expansion. They have become a pioneering work on resident daily life under the background of "Internet plus" environment. However, regardless of the great convenience for daily life, due to the platform enterprise itself usually has few cars, even has no cars, only by integrating social existing resources to provide service for passengers. The occurrence of adverse even malignant events acted by special car drivers are unable to be avoided efficiently in consequence of the independence from the platforms. Let's take Uber, which is in the limelight of the Chinese market, as an example. As one of the world's most famous and successful tailored taxi service app, its location is more than just a taxi applications, it's also " a high-end and more private travel program for passengers", which provides a good service for costumers by social private car resource integration without owning a car itself. But at the same time, people are suspecting of the current taxi-hailing platform operation mode because of the bad behaviors the drivers did. It is reported that, in December 2015, the Uber driver raped female 
passenger vicious incident happened in India, not only caused irreparable blow to the reputation of Uber, but also directly led to the government of New Delhi issued a ban on Uber. In July 2016, the British woman Anna Kealey got the driver's phone threats and intimidation just due to cancel an order, although Uber support staff quickly made a positive response, said they would immediately dismiss the pilot and investigate the incident, but the negative effect was difficult to eliminate. Except Uber, Didi, Kuaidi and other well-known platform operators also happened irregularities and even illegal operations of the driver, caused serious damage to the company's reputation, which affects their demand of the service market and profit.

Apparently, under the current marketing supervision mechanism, if the taxi drivers who provide the direct service for the passengers, couldn't be restrained, guided and inspired correctly in long time, the effort level and service quality will be hard to maintained on a high standard, which will be harmful to the development of the whole Internet taxi taking industry, platform enterprises and drivers will lose the opportunities of making profit. So, during the service operation process, how do the enterprises set up reasonable incentive mechanism and improve the drivers' participation enthusiasm and effort level to meet the passengers' demand will be the challenge and key point of managing the service supply chain effectively.

\section{Literature Review}

Cooperation between member enterprises or individuals through incentives to achieve coordination is an important research content in the field of enterprise management and academic circles, and reasonable contract design can achieve mutual benefit, that has become an important incentive way [1-2]. At present, according to the contract, especially on cost sharing contract mainly concentrated in the field of supply chain has achieved many results [3-7]. Cost sharing contract as an important way to achieve supply chain coordination, has been widespread concern and research [8-10].Lu Qihui [11] suppose that the market demand and service capacity are respectively related to the support level of service providers and service providers, and establish a service supply chain model based on cost sharing strategy; Gary Huaite [12] by establishing and solving the model found that in a monopoly or oligopoly market, only manufacturers and retailers between the cost sharing contract, it may be effective to maintain its market position; Hu Benyong, Lei Dong and Chen $\mathrm{Xu}$ [13] provides an option pricing method based on expected loss, that when the cost sharing and reputation sharing ratio to meet certain conditions, can achieve effective coordination of supply chain.

At the same time, it is worth noting that the current design of the supply chain contracts is based on the premise of a one-time transaction relationship between the supply chain members. In reality, however, members of the supply chain are usually a long-term and stable cooperative partnership. At this time, the time is an important factor among the members of the game, because the decision makers in a moment of optimal decision does not guarantee that the follow-up time is still optimal, so the members of the supply chain strategy is a function of time, the game between each other will become a differential game problem. Research on the strategy of differential game originated in the military struggle between the two sides of confrontation, at present in the field of management on the application includes investment decision-making, duopoly competition and cooperative advertising [14-19].

In the existing literature, the research on the cooperation and coordination between enterprises and individuals is more and more used to determine the reputation model, however, the random reputation is more common in the actual operation. The study of random reputation goes back to the research of Tapiero [20], which uses stochastic dynamic optimization theory to establish a stochastic reputation model of monopoly firms and finds the relationship between firm investment and risk appetite. Subsequently, Tapiero [21] extends the random reputation model To multi-enterprise scenarios. Marinelli [22] explained that the reason for the random reputation is that the reputation of the enterprise is affected by external factors. At the same time, Marinelli [22] pointed out that the source of enterprise reputation has random are: the "background", is a kind of additional types of interference, it is not affected by the popularity of existing products; the potential consumer uncertainty to the enterprise product knowledge; the enterprise advertising produce the effect of uncertainty. Similarly, in the taxi 
service supply chain based on the Internet, because of the political environment, human factors are uncertain to provide customers the service supply chain service awareness or supply chain members to level effects, which making the supply chain reputation inevitably randomness.

\section{Model}

Corporate reputation, as one of the most important intangible assets, is the comprehensive reflection of the behavior of corporate in the past [23]. The results of empirical research of service industry indicate that reputation is conducive to the creation and maintaining of customer trust resulting in the increase in successive purchase by loyal customers [24]. Hence, the demand of the Tailored Taxi Service System can be reflected by the function of the reputation of this service system, and the accumulation of this service system reputation depends on the efforts contributed by the platform enterprise and special car driver in their respective service.

It is assumed that the demand of logistics service $S(t)$ adopts the following specification:

$S(t)=\alpha E_{i}(\mathrm{t})+\beta E_{p}(\mathrm{t})+\theta R(\mathrm{t})-\gamma R^{2}(t)$

Where $E_{i}(\mathrm{t})$ and $E_{p}(\mathrm{t})$ respectively platform enterprise and the special car driver's effort level and $\alpha, \beta, \theta$ and $\gamma$ are positive parameters capturing the effect of the reputation on the demand.

The growth and accumulation of the reputation $R(t)$ of this service system depend on the efforts of supply members. The service integrator controls his efforts $E_{i}(\mathrm{t})$ while the service supplier controls his efforts $E p(t)$ during the cooperation in service.

The efforts of both parties during the service contribute to the accumulation of the reputation of this service system $R(t)$, which evolves according to the Nerlove and Arrow model ; that is,

$$
d R(t)=\left[\lambda_{i}(\mathrm{t}) E_{i}(t)+\lambda_{p}(\mathrm{t}) E_{p}(t)-\delta(\mathrm{t}) R(t)\right] \mathrm{dt}+\sigma(\mathrm{R}(\mathrm{t})) \mathrm{dz}(\mathrm{t}), R(0)=R_{0} \geq 0
$$

Where $\lambda i(t)>0$ and $\lambda p(t)>0$ reflecting the efficiency of the efforts of the integrator and the supplier, respectively. Since the reputation decays by time, $\delta(t)>0$, in fact, is the constant decay rate of this service system reputation caused by environment disturbance

Suppose the platform enterprise and the special car driver's reputation maintenance cost functions are:

$$
C_{i}(t)=\frac{\mu_{i}(t)}{2} E_{i}^{2}(t), C_{p}(t)=\frac{\mu_{p}(t)}{2} E_{p}^{2}(t)
$$

Where $a$ and $b$ are the reputation maintenance cost coefficients of the platform enterprise and the special car driver, respectively $\mathrm{c}$ and $\mathrm{d}$ represent the reputation maintenance costs of the platform enterprise and the special car driver, all of which are increasing functions of effort level and show strict convexity.

Let $D(t)$ be the rate of cost that the platform enterprise will share with the special car driver, which ranges from zero (the platform enterprise will not share the cost) to one (the platform enterprise pays the full cost). Let $\rho$ denote the discount rate of both parties. $\pi_{i}(t)$ and $\pi_{p}(t)$ represent the profit margin of the platform enterprise and the special car driver, respectively. Consider

$$
\begin{aligned}
& \max \left\{J_{i}\left(\mathrm{R}_{0}\right)=E \int_{0}^{\infty} e^{-\rho t}\left\{\pi_{i}(t)\left[\alpha E_{i}(\mathrm{t})+\beta R(\mathrm{t})+\theta R(\mathrm{t})-\gamma R^{2}(t)\right]-\frac{\mu_{i}(t)}{2} E_{i}^{2}(t)-\frac{\mu_{p}(t)}{2} D(\mathrm{t}) E_{p}^{2}(t)\right\} d t\right\} \\
& \max \left\{J_{p}\left(\mathrm{R}_{0}\right)=E \int_{0}^{\infty} e^{-\rho t}\left\{\pi_{p}(t)\left[\alpha E_{i}(\mathrm{t})+\beta R(\mathrm{t})+\theta R(\mathrm{t})-\gamma R^{2}(t)\right]-\frac{\mu_{p}(t)}{2} E_{p}^{2}(t)[1-D(t)]\right\} d t\right\}
\end{aligned}
$$

To recapitulate, (1) to (4) define a differential game with two players, three control variables $E i(t)$, $E p(t)$, and $D(t)$, and one state variable $R(t)$. The controls are constrained by $E i(t) \geq 0, E p(t) \geq 0,0 \leq$ $D(t) \leq 1$. The state constraint $R(t) \geq 0$ is automatically satisfied.

The optimal decisions of both parties are decided by a feedback game, so that they are all functions of reputation and time. Let $I(R(t), t)$ and $P(R(t), t)$ be the decisions of the platform enterprise and the special car driver, respectively. To simplify the model, it is assumed that all the parameters in the model, $\mu_{i}(t), \mu_{p}(t), \lambda_{i}(t), \lambda_{p}(t), \delta(t), \theta(t), \pi_{i}(t)$ and $\pi_{p}(t)$ are constants and have nothing to do with time, 
which means the players are in the same game in different time horizons. Thus, the differential games can be changed to static games [51]. The decisions of both parties can be defined as $I(R(t))$ and $P(R(t))$. In the following model, $R(t), E_{i}(t), E_{p}(t)$ and $D(t)$ are simplified as $R, E i, E p$, and $D$.

\section{Stackelberg Equilibrium with Cost Sharing Contract}

In this section, we apply a cost sharing contract to realize the coordination of special car driver in a Stackelberg game with the platform enterprise as the leader of the game and the provider as the follower. Firstly, the integrator decides the optimal efforts $E i$ and the sharing rate $D$. Then, the functional service provider determines his optimal efforts after observing the strategy of the integrator. The optimal decisions of both parties are the results of Stackelberg equilibrium.

Theorem 1. If the feasible solutions to the following constraint equations ( $\left.p_{1}, p_{2}, p_{3}, q_{1}, q_{2}, q_{3}\right)$ exist, the optimal decisions of both parties can be derived after adopting the cost sharing contract in platform enterprise:

$$
\left\{\begin{array}{l}
\frac{2 \lambda_{i}^{2} p_{1}^{2}}{\mu_{i}}+\frac{\lambda_{p}^{2}\left(2 p_{1}+q_{1}\right)^{2}}{2 \mu_{p}}-\left(\pi_{i} \gamma+2 \delta p_{1}\right)-\rho p_{1}=0 \\
\pi_{i} \theta-(\delta+\rho) p_{2}+\frac{2 \lambda_{i} p_{1}\left(\alpha \pi_{i}+\lambda_{i} p_{2}\right)}{\mu_{i}}+\frac{\lambda_{p}\left(2 p_{1}+q_{1}\right)\left[\lambda_{p}\left(2 p_{2}+q_{2}\right)+\beta\left(2 \pi_{i}+\pi_{p}\right)\right]}{2 \mu_{p}}=0 \\
\frac{\left(\alpha \pi_{i}+\lambda_{i} p_{2}\right)^{2}}{2 \mu_{i}}+\frac{\left[\lambda_{p}\left(2 p_{2}+q_{2}\right)+\beta\left(2 \pi_{i}+\pi_{p}\right)\right]^{2}}{8 \mu_{p}}+p_{1} \sigma^{2}-\rho p_{3}=0 \\
\frac{4 \lambda_{i}^{2} p_{1} q_{1}}{\mu_{i}}+\frac{\lambda_{p}^{2} q_{1}\left(2 p_{1}+q_{1}\right)}{\mu_{p}}-\left[\pi_{p} \gamma+(2 \delta+\rho) q_{1}\right]=0 \\
\pi_{p} \theta-(\delta+\rho) q_{2}+\frac{2 \lambda_{i}\left[p_{1}\left(\alpha \pi_{p}+\lambda_{i} q_{2}\right)+q_{1}\left(\alpha \pi_{i}+\lambda_{i} p_{2}\right)\right]}{\mu_{i}} \\
\quad+\frac{2 \lambda_{p} q_{1}\left[\lambda_{p}\left(2 p_{2}+q_{2}\right)+\beta\left(2 \pi_{i}+\pi_{p}\right)\right]+2 \lambda_{p}\left(2 p_{1}+q_{1}\right)\left(\lambda_{p} q_{2}+\beta \pi_{p}\right)}{4 \mu_{p}}=0 \\
\frac{\left(\alpha \pi_{i}+\lambda_{i} p_{1}\right)\left(\alpha \pi_{p}+\lambda_{i} q_{2}\right)}{\mu_{i}}+\frac{\left(\lambda_{p} q_{2}+\beta \pi_{p}\right)\left[\left(2 p_{2}+q_{2}\right)+\beta\left(2 \pi_{i}+\pi_{p}\right)\right]}{4 \mu_{p}}+q_{1} \sigma^{2}(\mathrm{R})-\rho q_{3}=0
\end{array}\right.
$$

The optimal decisions of the platform enterprise and the special car driver in the Stackelberg equilibrium with the platform enterprise as the leader can be represented as

$$
\begin{aligned}
& E_{i}^{*}=\frac{\alpha \pi_{i}+\lambda_{i}\left(2 p_{1}^{*} R+p_{2}^{*}\right)}{\mu_{i}} \\
& E_{p}=\frac{\beta\left(2 \pi_{i}+\pi_{p}\right)+\lambda_{p}\left[\left(4 p_{1}^{*}+2 q_{1}^{*}\right) R+\left(2 p_{2}^{*}+q_{2}^{*}\right)\right]}{2 \mu_{p}} \\
& D^{*}=\frac{\beta\left(2 \pi_{i}-\pi_{p}\right)+\lambda_{p}\left(\left(4 p_{1}^{*}-2 q_{1}^{*}\right) R+\left(2 p_{2}^{*}-q_{2}^{*}\right)\right)}{\beta\left(2 \pi_{i}+\pi_{p}\right)+\lambda_{p}\left(\left(4 p_{1}^{*}+2 q_{1}^{*}\right) R+\left(2 p_{2}^{*}+q_{2}^{*}\right)\right)}
\end{aligned}
$$

Proof. Given that the special car driver announced decisions $E i$ and $D$, the provider faces a maximization problem where the profit function must satisfy the HJB equation

$$
\rho V_{p}(R)=\operatorname{Max}_{E_{p} \geq 0}\left\{\pi_{p}\left(\alpha \mathrm{E}_{i}+\beta E_{p}(\mathrm{t})+\theta R(\mathrm{t})-\gamma R^{2}(t)\right)-\frac{\mu_{p}}{2}(1-D) E_{p}^{2}+V_{p}^{\prime}(R)\left[\lambda_{i} E_{i}+\lambda_{p} E_{p}-\delta R\right]+\frac{\sigma^{2}(\mathrm{R}) \mathrm{V}_{p}^{\prime \prime}(\mathrm{R})}{2}\right\}
$$

Maximization on the right side of (10) yields

$$
E_{p}=\frac{\lambda_{p} V_{p}^{\prime}(R)+\beta \pi_{p}}{\mu_{p}(1-D)}
$$

The platform enterprise, as the leader of game, can forecast the decision of the special car driver, and then the leader HJB equation is 


$$
\rho V_{i}(R)=\operatorname{Max}_{E_{i} \geq 0}\left\{\pi_{i}\left(\alpha \mathrm{E}_{i}+\beta E_{p}(\mathrm{t})+\theta R(\mathrm{t})-\gamma R^{2}(t)\right)-\frac{\mu_{i}}{2} E_{i}^{2}-\frac{\mu_{p}}{2} D E_{p}^{2}+V_{i}^{\prime}(R)\left[\lambda_{i} E_{i}+\lambda_{p} E_{p}-\delta R\right]+\frac{\sigma^{2}(\mathrm{R}) \mathrm{V}_{i}^{\prime \prime}(\mathrm{R})}{2}\right\}
$$

Insert (11) into (12) and obtain the efforts and sharing rate as

$$
\begin{aligned}
E_{i} & =\frac{\alpha \pi_{i}+\lambda_{i} V_{i}^{\prime}(R)}{\mu_{i}} \\
D & =\frac{\beta\left(2 \pi_{i}-\pi_{p}\right)+\lambda_{p}\left(2 V_{i}^{\prime}(R)-V_{p}^{\prime}(R)\right)}{\beta\left(2 \pi_{i}+\pi_{p}\right)+\lambda_{p}\left(2 V_{i}^{\prime}(R)+V_{p}^{\prime}(R)\right)}
\end{aligned}
$$

Insert (11), (13), and (14) into (10) and (12) to obtain

$$
\begin{aligned}
\rho V_{i}(R)= & {\left[\pi_{i}(\theta-\gamma R)-\delta V_{i}^{\prime}(R)\right] R+\frac{\left[\alpha \pi_{i}+\lambda_{i} V_{i}^{\prime}(R)\right]^{2}}{2 \mu_{i}}+\frac{\left[\beta\left(2 \pi_{i}+\pi_{p}\right)+\lambda_{p}\left(2 V_{i}^{\prime}(R)+V_{p}^{\prime}(R)\right)\right]^{2}}{8 \mu_{p}} } \\
& +\frac{\sigma^{2}(\mathrm{R}) \mathrm{V}_{i}^{\prime \prime}(\mathrm{R})}{2} \\
\rho V_{p}(R)= & {\left[\pi_{i}(\theta-\gamma R)-\delta V_{i}^{\prime}(R)\right] R+\frac{\left(\alpha \pi_{i}+\lambda_{i} V_{i}^{\prime}(R)\right)\left(\alpha \pi_{p}+\lambda_{i} V_{p}^{\prime}(R)\right)}{\mu_{i}} } \\
& +\frac{\left(\beta \pi_{p}+\lambda_{p} V_{p}^{\prime}\right)\left[\beta\left(2 \pi_{i}+\pi_{p}\right)+\lambda_{p}\left(2 V_{i}^{\prime}(R)+V_{p}^{\prime}(R)\right)\right]}{4 \mu_{p}}+\frac{\sigma^{2}(\mathrm{R}) \mathrm{V}_{i}^{\prime \prime}(\mathrm{R})}{2}
\end{aligned}
$$

We conjecture that the solutions of (15) and (16) will be quadratic $V_{i}(R)=p_{1} R^{2}+p_{2} R+p_{3}, V_{p}(R)=q_{1} R^{2}+q_{2} R+q_{3}$

in which $p_{1}, p_{2}, p_{3}$ and $q_{1}, q_{2}, q_{3}$ are all constants. We substitute $\operatorname{Vi}(R), \operatorname{Vp}(R)$ and their derivatives from (16) into (15) to obtain

$$
\begin{aligned}
\rho\left(p_{1} R^{2}+p_{2} R+p_{3}\right)= & {\left[\pi_{i}(\theta-\gamma R)-\delta\left(2 p_{1} R+p_{2}\right)\right] R+\frac{\left[\alpha \pi_{i}+\lambda_{i}\left(2 p_{1} R+p_{2}\right)\right]^{2}}{2 \mu_{i}} } \\
& +\frac{\left[\beta\left(2 \pi_{i}+\pi_{p}\right)+\lambda_{p}\left[\left(4 p_{1}+2 q_{1}\right) R+\left(2 p_{2}+q_{2}\right)\right]\right]^{2}}{8 \mu_{p}}+p_{1} \sigma^{2} \\
\rho\left(q_{1} R^{2}+q_{2} R+q_{3}\right)= & {\left[\pi_{i}(\theta-\gamma R)-\delta\left(2 p_{1} R+p_{2}\right)\right] R+\frac{\left(\alpha \pi_{i}+\lambda_{i}\left(2 p_{1} R+p_{2}\right)\right)\left(\alpha \pi_{p}+\lambda_{i}\left(2 q_{1} R+q_{2}\right)\right)}{\mu_{i}} } \\
+ & \frac{\left(\beta \pi_{p}+\lambda_{p}\left(2 q_{1} R+q_{2}\right)\right)\left[\beta\left(2 \pi_{i}+\pi_{p}\right)+\lambda_{p}\left(\left(4 p_{1}+2 q_{1}\right) R+\left(2 p_{2}+q_{2}\right)\right)\right]}{4 \mu_{p}}+q_{1} \sigma^{2}(\mathrm{R})
\end{aligned}
$$

Equations (18) and (19) both satisfy for all $R \geq 0$.Thus, we can find out that a set of values for $\left(p_{1}^{*}, p_{2}^{*}, p_{3}^{*}, q_{1}^{*}, q_{2}^{*}, q_{3}^{*}\right)$, which can result in the maximization of the profits, is the solution for the following equations:

$$
\begin{aligned}
& \rho \rho p_{1}=\frac{2 \lambda_{i}^{2} p_{1}^{2}}{\mu_{i}}+\frac{\lambda_{p}^{2}\left(2 p_{1}+q_{1}\right)^{2}}{2 \mu_{p}}-\left(\pi_{i} \gamma+2 \delta p_{1}\right) \\
& \rho p_{2}=\pi_{i} \theta-\delta p_{2}+\frac{2 \lambda_{i} p_{1}\left(\alpha \pi_{i}+\lambda_{i} p_{2}\right)}{\mu_{i}}+\frac{\lambda_{p}\left(2 p_{1}+q_{1}\right)\left[\lambda_{p}\left(2 p_{2}+q_{2}\right)+\beta\left(2 \pi_{i}+\pi_{p}\right)\right]}{2 \mu_{p}} \\
& \rho p_{3}=\frac{\left(\alpha \pi_{i}+\lambda_{i} p_{2}\right)^{2}}{2 \mu_{i}}+\frac{\left[\lambda_{p}\left(2 p_{2}+q_{2}\right)+\beta\left(2 \pi_{i}+\pi_{p}\right)\right]^{2}}{8 \mu_{p}}+p_{1} \sigma^{2} \\
& \left\{\rho q_{1}=\frac{4 \lambda_{i}^{2} p_{1} q_{1}}{\mu_{i}}+\frac{\lambda_{p}^{2} q_{1}\left(2 p_{1}+q_{1}\right)}{\mu_{p}}-\left(\pi_{p} \gamma+2 \delta q_{1}\right)\right. \\
& \rho q_{2}=\pi_{p} \theta-\delta q_{2}+\frac{2 \lambda_{i}\left[p_{1}\left(\alpha \pi_{p}+\lambda_{i} q_{2}\right)+q_{1}\left(\alpha \pi_{i}+\lambda_{i} p_{2}\right)\right]}{\mu_{i}} \\
& +\frac{2 \lambda_{p} q_{1}\left[\lambda_{p}\left(2 p_{2}+q_{2}\right)+\beta\left(2 \pi_{i}+\pi_{p}\right)\right]+2 \lambda_{p}\left(2 p_{1}+q_{1}\right)\left(\lambda_{p} q_{2}+\beta \pi_{p}\right)}{4 \mu_{p}} \\
& \rho q_{3}=\frac{\left(\alpha \pi_{i}+\lambda_{i} p_{1}\right)\left(\alpha \pi_{p}+\lambda_{i} q_{2}\right)}{\mu_{i}}+\frac{\left(\lambda_{p} q_{2}+\beta \pi_{p}\right)\left[\left(2 p_{2}+q_{2}\right)+\beta\left(2 \pi_{i}+\pi_{p}\right)\right]}{4 \mu_{p}}+q_{1} \sigma^{2}(\mathrm{R})
\end{aligned}
$$


The optimal profits of the platform enterprise and special car driver can be represented as

$$
\begin{aligned}
& V_{i}^{*}(R)=p_{1}^{*} R^{2}+p_{2}^{*} R+p_{3}^{*} \\
& V_{p}^{*}(R)=q_{1}^{*} R^{2}+q_{2}^{*} R+q_{3}^{*}
\end{aligned}
$$

Substituting the derivative of $\operatorname{Vi}(R)$ from (21) into (13), the optimal efforts of special car driver can be represented as

$$
E_{i}^{*}=\frac{\alpha \pi_{i}+\lambda_{i}\left(2 p_{1}^{*} R+p_{2}^{*}\right)}{\mu_{i}}
$$

Substituting the derivatives of $V i(R)$ and $V p(R)$ from(21) and (22) into (14), the optimal sharing rate can be represented as

$$
D^{*}=\frac{\beta\left(2 \pi_{i}-\pi_{p}\right)+\lambda_{p}\left(\left(4 p_{1}^{*}-2 q_{1}^{*}\right) R+\left(2 p_{2}^{*}-q_{2}^{*}\right)\right)}{\beta\left(2 \pi_{i}+\pi_{p}\right)+\lambda_{p}\left(\left(4 p_{1}^{*}+2 q_{1}^{*}\right) R+\left(2 p_{2}^{*}+q_{2}^{*}\right)\right)}
$$

Substituting the derivative of (22) with respect to $\mathrm{R}$ and (24) into (11), the optimum special car driver effort level can be represented as

$$
E_{p}=\frac{\beta\left(2 \pi_{i}+\pi_{p}\right)+\lambda_{p}\left[\left(4 p_{1}^{*}+2 q_{1}^{*}\right) R+\left(2 p_{2}^{*}+q_{2}^{*}\right)\right]}{2 \mu_{p}}
$$

\section{Cooperative Game}

In this part, we try to discuss the game situation that the platform enterprise implements the cost-sharing incentive strategy for the special car driver, and the two cooperate and decide together. Cooperative game can be used as a benchmark, a target to be achieved by non-cooperative game. Cooperative game means that the two non-cooperative game under the decision-makers into a cooperative game under the decision-makers. The effectiveness of the cost sharing incentive scheme is proved by comparison with the non-cooperative equilibrium results.

When the platform enterprise and the special car driver to cooperate in the game, the two sides will maximize the profit of the supply chain system as the goal. At this time the supply chain objective function as:

$$
\max \left\{J\left(\mathrm{R}_{0}\right)=J_{i}\left(\mathrm{R}_{0}\right)+J_{p}\left(\mathrm{R}_{0}\right)=E \int_{0}^{\infty} e^{-\rho t}\left\{\left(\pi_{i}+\pi_{P}\right)\left(\alpha E_{i}+\beta E_{p}(\mathrm{t})+\theta R(\mathrm{t})-\gamma R^{2}(t)\right)-\frac{\mu_{i}}{2} E_{i}^{2}-\frac{\mu_{p}}{2} E_{p}^{2}\right\} d t\right\}
$$

The optimal profit function $V(R)$ must satisfy the HJB equation:

$$
\rho V(R)=\operatorname{Max}_{\substack{E_{i} \geq 0 \\ E_{p} \geq 0}}\left\{\left(\left(\pi_{i}+\pi_{p}\right)\left(\alpha \mathrm{E}_{i}+\beta E_{p}(\mathrm{t})+\theta R(\mathrm{t})-\gamma R^{2}(t)\right)-\frac{\mu_{i}}{2} E_{i}^{2}-\frac{\mu_{p}}{2} E_{p}^{2}+V^{\prime}(R) \mathrm{R}+\frac{\sigma^{2}(\mathrm{R}) V^{\prime \prime}(\mathrm{R})}{2}\right\}\right.
$$

Equations (27) are concave in $E i$ and $E p$, yielding the unique effort level of both parties

$$
E_{i}=\frac{\alpha\left(\pi_{i}+\pi_{p}\right)+\lambda_{i} V^{\prime}(R)}{\mu_{i}}, E_{p}=\frac{\beta\left(\pi_{i}+\pi_{p}\right)+\lambda_{p} V^{\prime}(R)}{\mu_{p}}
$$

Substitute $E i$ and $E p$ into (27) to obtain

$$
\begin{aligned}
\rho V(R)= & -\left(\pi_{i}+\pi_{p}\right) \gamma R^{2}+\left[\left(\pi_{i}+\pi_{p}\right) \theta-\delta V^{\prime}(R)\right] R+\frac{\left[\alpha\left(\pi_{i}+\pi_{p}\right)+\lambda_{i} V^{\prime}(R)\right]^{2}}{2 \mu_{i}} \\
& +\frac{\left[\beta\left(\pi_{i}+\pi_{p}\right)+\lambda_{p} V^{\prime}(R)\right]^{2}}{2 \mu_{p}}+\frac{\sigma^{2}(\mathrm{R}) V^{\prime \prime}(\mathrm{R})}{2}
\end{aligned}
$$

We conjecture that the solutions to (29) will be quadratic

$$
V(R)=l_{1} R^{2}+l_{2} R+l_{3}
$$

in which $l_{1}, l_{2}$ are constants. We substitute $V(R)$ and their derivatives from(30) into (29) to obtain

$$
\begin{aligned}
\rho\left(l_{1} R^{2}+l_{2} R+l_{3}\right)= & {\left[-\left(\pi_{i}+\pi_{p}\right) \gamma-2 \delta l_{1}+\frac{2 \lambda_{i}^{2} l_{1}}{\mu_{i}}+\frac{2 \lambda_{p}^{2} l_{1}^{2}}{\mu_{p}}\right] \mathrm{R}^{2}+\left[\left(\pi_{i}+\pi_{p}\right) \theta-\delta l_{2}+\frac{2 \lambda_{i} l_{1}\left[\alpha\left(\pi_{i}+\pi_{p}\right)+l_{2} \lambda_{i}\right]}{\mu_{i}}\right.} \\
& \left.+\frac{2 \lambda_{p} l_{1}\left[\alpha\left(\pi_{i}+\pi_{p}\right)+l_{2} \lambda_{p}\right]}{\mu_{p}}\right] \mathrm{R}+\frac{\left[\alpha\left(\pi_{i}+\pi_{p}\right)+\lambda_{i} l_{2}\right]^{2}}{2 \mu_{i}}+\frac{\left[\beta\left(\pi_{i}+\pi_{p}\right)+\lambda_{p} l_{2}\right]^{2}}{2 \mu_{p}}+l_{1} \sigma^{2}
\end{aligned}
$$


Equations (12) and (13) both satisfy for all $R \geq 0$. Thus,

We can find out that a set of values for $\left(l_{1}^{*}, l_{2}^{*}, l_{3}^{*}\right)$, which can result in the maximization of the profits, is the solution for the following equations:

$$
\left\{\begin{array}{l}
\rho l_{1}=-\left(\pi_{i}+\pi_{p}\right) \gamma-2 \delta l_{1}+\frac{2 \lambda_{i}^{2} l_{1}}{\mu_{i}}+\frac{2 \lambda_{p}^{2} l_{1}^{2}}{\mu_{p}} \\
\rho l_{2}=\left(\pi_{i}+\pi_{p}\right) \theta-\delta l_{2}+\frac{2 \lambda_{i} l_{1}\left[\alpha\left(\pi_{i}+\pi_{p}\right)+l_{2} \lambda_{i}\right]}{\mu_{i}}+\frac{2 \lambda_{p} l_{1}\left[\alpha\left(\pi_{i}+\pi_{p}\right)+l_{2} \lambda_{p}\right]}{\mu_{p}} \\
\rho l_{3}=\frac{\left[\alpha\left(\pi_{i}+\pi_{p}\right)+\lambda_{i} l_{2}\right]^{2}}{2 \mu_{i}}+\frac{\left[\beta\left(\pi_{i}+\pi_{p}\right)+\lambda_{p} l_{2}\right]^{2}}{2 \mu_{p}}+l_{1} \sigma^{2}
\end{array}\right.
$$

Then, the optimal profits of the platform enterprise and special car driver can be represented as

$$
V^{*}(R)=l_{1}^{*} R^{2}+l_{2}^{*} R+l_{3}^{*}
$$

Substituting the derivatives of $V i(R), V p(R)$ obtained from (33) into (28), the optimal effort level of both parties can be represented as

$$
\begin{aligned}
& E_{i}^{*}=\frac{\alpha\left(\pi_{i}+\pi_{p}\right)+\lambda_{i}\left(2 l_{1}^{*} R+l_{2}^{*}\right)}{\mu_{i}} \\
& E_{p}^{*}=\frac{\beta\left(\pi_{i}+\pi_{p}\right)+\lambda_{p}\left(2 l_{1}^{*} R+l_{2}^{*}\right)}{\mu_{p}}
\end{aligned}
$$

\section{The Influence of the Best Effort of Two Parties on Reputation under Stackelberg Game}

From Part 4, it can be seen that the profits of the two channels are related to the reputation of the platform enterprise, while the reputation is a random variable. Therefore, it is necessary to study the expectation and variance of reputation. If the software the platform enterprise invests the optimal national advertisement and the special car driver puts the best effort level, solves the reputation expectation and the variance. Substitute the balance of national advertising and local advertising in Part 4 into (2) to obtain

$$
d R(t)=[\Omega-\chi R(t)] \mathrm{dt}+\sigma(R(\mathrm{t})) \mathrm{dz}(\mathrm{t})
$$

In which $\Omega=\frac{\lambda_{i}\left(\alpha \pi_{i}+\lambda_{i}^{2} p_{2}^{*}\right)}{\mu_{i}}+\frac{\lambda_{p}\left[\beta\left(2 \pi_{i}+\pi_{p}\right)+\left(2 p_{2}^{*}+q_{2}^{*}\right)\right]}{2 \mu_{p}} \chi=\frac{2 \lambda_{1}^{2} p_{1}^{*}}{\mu_{i}}+\frac{\lambda_{p}^{2}\left[\left(4 p_{1}^{*}+2 q_{1}^{*}\right)\right]}{2 \mu_{p}}-\delta$ are constants.

Under the optimal effort of Stackelberg game, the expectation and variance of the platform enterprise's reputation are:

$$
\begin{aligned}
& E\left[R^{2}(t)\right]=\frac{\left(2 \Omega+\sigma^{2}\right)\left(\Omega e^{2 \chi t}+2 \chi R_{0} e^{\chi t}-2 \Omega e^{\chi t}+\Omega-2 \chi R_{0}\right)}{2 \chi^{2} e^{2 \chi t}}+R_{0}^{2} e^{-2 \chi t} \\
& D[R(t)]=\frac{\sigma^{2}\left[\Omega+2 \chi R_{0} e^{-\chi t}-2 \Omega e^{-\chi t}+\left(\Omega-2 \chi R_{0}\right) e^{-2 \chi t}\right]}{2 \chi^{2}}
\end{aligned}
$$

Proof: rewrite (36) as random differential form

$$
R(t)=R_{0}+\int_{0}^{t}(\Omega-\chi R(t)) d s+\int_{0}^{t} \sigma(R(\mathrm{~s})) d z(s)
$$

Solve the expectation of $\mathrm{R}(\mathrm{t})$

$$
E[R(t)]=R_{0}+\int_{0}^{t}(\Omega-\chi E(R(t))) d s
$$

Equation a can be transformed into an ordinary differential equation with initial condition $\mathrm{E}[\mathrm{R}(0)]=$ Ro

$E[R(t)]=\frac{\Omega}{\chi}+e^{\chi t}\left(R_{0}-\frac{\Omega}{\chi}\right)$

Use Ito formula on (36)

$$
\begin{aligned}
& d R^{2}(t)=\left[2 R(\Omega-\chi R)+\sigma^{2} R\right] d t+2 R \sigma \sqrt{R} d z \\
& E\left[R^{2}(t)\right]=R_{0}^{2}+\int_{0}^{t}\left[\left(2 \Omega+\sigma^{2}\right) E(R(\mathrm{~s}))-2 \chi E\left(R^{2}(\mathrm{~s})\right)\right] d s
\end{aligned}
$$

Rewrite (42) into the form of random differentials and Solve the expectation 


$$
\begin{aligned}
& d E\left[R^{2}(t)\right] / d t=\left(2 \Omega+\sigma^{2}\right)\left[\frac{\Omega}{\chi}+e^{\chi t}\left(R_{0}-\frac{\Omega}{\chi}\right)\right]-2 \chi E\left(R^{2}\right) \\
& E\left[R^{2}(t)\right]=\frac{\left(2 \Omega+\sigma^{2}\right)\left(\Omega e^{2 \chi t}+2 \chi R_{0} e^{\chi t}-2 \Omega e^{\chi t}+\Omega-2 \chi R_{0}\right)}{2 \chi^{2} e^{2 \chi t}}+R_{0}^{2} e^{-2 \chi t}
\end{aligned}
$$

Study the relationship between expectation and variance, Confidence interval is used to describe the real reputation, At the $95 \%$ confidence level, the confidence interval for reputation is

$$
(E[R(t)]-1.96 \sqrt{D[R(t)]}, E[R(t)]-1.96 \sqrt{D[R(t)]})
$$

\section{The Expectation and Variance Properties of StackelbergGame}

When $\sigma=0, \lim _{t \rightarrow \infty} E\left[R^{2}(t)\right]=\lim _{t \rightarrow \infty}(E[R(t)])^{2}, \lim _{t \rightarrow \infty} D[R(t)]=0$;

In the Stackelberg game with optimal input, when $\chi>\Omega / R_{0}$, the expectations for reputation is decreasing over time, but always greater than $\Omega / \chi$;

When $\chi \leq \Omega / R_{0}$, the expectations for reputation is decreasing over time, but always less than $\Omega / \chi$;

Proof: The conclusion of (a) is obvious, now prove the conclusion of (b).

The expectation and variance of reputation in Section 4 to $\mathrm{R}$ derivative

$$
\begin{aligned}
& \left.d E[R(t)] / d t=-\chi e^{\chi t}\left(R_{0}-\frac{\Omega}{\chi}\right)\right] \\
& \frac{d D[R(t)]}{d t}=\frac{\sigma^{2} e^{-\chi t}\left[\Omega\left(1-e^{-\chi t}\right)-\chi R_{0}\left(1-2 e^{-\chi t}\right)\right]}{\chi}
\end{aligned}
$$

So, $\chi=\Omega / R_{0}$ As a threshold, we got (b).

\section{The Influence of the Best Effort Degree of Two Parties on reputation under Cooperative Game}

When the two Parties cooperate in the game and use the optimal advertising investment, we study the reputation expectations and variance. Substituting (34) and (35) into (2)

$d R(t)=[\bar{\Omega}-\bar{\chi} R(t)] \mathrm{d} \mathrm{t}+\sigma(R(\mathrm{t})) \mathrm{dz}(\mathrm{t})$

In which $\bar{\Omega}=\frac{\alpha \lambda_{i}\left(\pi_{i}+\pi_{p}\right)+l_{2} \lambda_{1}^{2}}{\mu_{i}}+\frac{\beta \lambda_{p}\left(\pi_{i}+\pi_{p}\right)+l_{2} \lambda_{p}^{2}}{\mu_{p}} \bar{\chi}=\delta-\frac{2 \lambda_{1}^{2} l_{1}^{*}}{\mu_{i}}-\frac{2 l_{1}^{*} \lambda_{p}^{2}}{\mu_{p}}$ are constants, using the stochastic breeze equation theory, the expectations and variance of the platform enterprise are given below.

In the cooperative game with optimal advertising investment, the platform enterprise's expectations for reputation

$$
E[R(t)]=\frac{\bar{\Omega}}{\bar{\chi}}+e^{\chi t}\left(R_{0}-\frac{\bar{\Omega}}{\bar{\chi}}\right)
$$

The variance of the reputation of the platform enterprise is:

$$
D[R(t)]=\frac{\sigma^{2}\left[\bar{\Omega}+2 \bar{\chi} R_{0} e^{-\chi t}-2 \bar{\Omega} e^{-\chi t}+\left(\bar{\Omega}-2 \bar{\chi} R_{0}\right) e^{-2 \chi t}\right]}{2 \bar{\chi}^{2}}
$$

\section{Summary}

Given the long-term relationship among members in the tailored taxi service system, we take time and reputation into consideration as the main factors in a dynamic differential model consisting of one platform enterprise and one special car driver. The results indicate the autonomy of this service system and the effectiveness of cost sharing contract on the coordination between the two players.

However, we simplify the parameters, the expression form of which may be various in reality resulting in different forms of solutions, which is a further research subject for future work. At the same time, research on the tailored taxi service system consisting of multi- platform enterprises and multispecial car drivers will make great sense in this field. 


\section{Acknowledgments}

This research was supported by the NSFC Major Program(Grant no.71090404/71090400) and NSFC General Program(Grant nos.71272045 and 71101107).

\section{References}

[1] Cachon G P, Supply Chain Coordination with Contracts, in: A.G. deKok, S.C. Graves (Eds.), Supply Chain Management: Design, Coordination and Operation, Elsevier, Amsterdam, 2003, pp. 229-340.

[2] Yang Y, Liu S H. Commitment Cost and Contractual Coordination Machanism of Supply Chain under OEM [J]. Systems Engineering, 2011,29(2):94-98.

[3] Wang X H, Wang X Y, Su Y S. Wholesale-price contract of Supply Chain with Information Gathering [J]. Applied Mathematical Modeling, 2013, 37(6): 3848-3860.

[4] Karakaya S, Bakal I S. Joint Quantity Flexibility for Multiple products in a Decentralized Supply Chain [J]. Computers \& Industrial Engineering, 2013, 64(2): 696-707.

[5] Xiong H C, Chen B T, Xie J X. A Composite Contract Based on Buy Back and Quantity Flexibility Contracts [J]. European Journal of Operational Research, 2011, 210(3): 559-567.

[6] Omkar D P D. Supply Chain Coordination Using Revenue-Dependent Revenue Sharing Contracts [J]. Omega, 2013, 41(4): 780-796.

[7] Liang L, Wang X H, Gao J G. An Option Contract Pricing Model of Relief Material Supply Chain [J]. Omega, 2012, 40(5): 594-600.

[8] Leng M M, Mahmut P. Game-theoretic Analyses of Decentralized Assembly Supply Chains: Non-cooperative Equilibria vs. Coordination with Cost-sharing Contracts [J]. European Journal of Operational Research, 2010, 204(1): 96-104.

[9] Kunter M. Coordination via Cost and Revenue sharing in Manufacturer-retailer Channels [J]. European Journal of Operational Research, 2012, 216(2): 477-486.

[10] Tsao Y C, Sheen G J. Effects of Promotion Cost Sharing Policy with The Sales Learning Curve on Supply Chain Coordination [J]. Computers \& Operations Research, 2012, 39(8): 1872-1878.

[11] Lu Q H. Coordinate with Cost Sharing Strategy for Service Supply Chain [J]. Control and Decision, 2011, 26(11): 1649-1653.

[12] Gary Huaite. External Quality Cost Sharing Sontracts in Supply Chains[D].Northwestern University.2007.

[13] Hu B Y, Lei D, Chen X. Supply Chain Option Sale-surety Contract Based on Revenue Sharing and Effort Cost partaking [J]. Journal of Industrial Engineering and Engineering Management,2010, 3 : 33-38.

[14] Navas J, Marín-Solano J. Interactions between Government and Firms: a Differential Game Approach [J]. Annals of Operations Research, 2008, 158: 47-61.

[15] Nie J J, Xiong Z K. Differential Game Models of Vertical Cooperative Advertising with Multiple Advertisingmedia [J]. Journal of Management Sciences in China, 2010, 13(5):1-10.

[16] Chen X, Zhang J, Zhang R. The Stackelberg Differential Game Model and Intergovermental Cooperation Design of Real Estate Market [J]. Systems Engineering-Theory \& Practice, 2011, 31(7):1293-1302.

[17]Cao G H,Jiang D L,Tang R J. Dynamic Optimal Decision Based on Watershed EcoCompensationApplica tion of Di fferential Game Theo ry [J]. Systems Engineering, 2011, 29(11): 63-70.

[18] Xu H T, Zhou X W. Optimal Power Control in Cooperative Relay Networks Based on a Differential Game [J].ETRI Journal, 2014, 36(2):280-284.

[19] Huang S J,Ren Y L,Sun R,et al. Stochastic Differential Game Models of Vertical Cooperative Mitigation in Duopoly Electricity Market [J]. Chinese Journal of Management Science, 2014, 22(2):101-111.

[20] Tapiero, Charles S. Optimum advertising and Goodwill under Uncertainty. Operations Research 
1978, 26(3): 450-463.

[21] Tapiero, Charles S. A Generalization of The Nerlove-Arrow Model to Multi-firms Advertising under Uncertainty. Management Science 1979, 25(9): 907-915.

[22] Marinelli, Carlo. The Stochastic Goodwill Problem. European Journal of Operational Research 2007, 76(1): 389-404.

[23] C. J. Fombrun,V. Rindova, "Who's tops and who decides The social construction of corporate $r$ eputations," Working Paper, Stern School of Business, New York University, 1996.

[24] N. Nha and L. Gaston, "Corporate image and corporate reputation in customers. Retention decisi ons in services," Journal of Retailing and Consumer Services, no. 8, pp. 227-2361, 2001. 